\title{
A child with Hallermann-Streiff syndrome; as an infrequent cause of hypotrichosis and cataract
}

\author{
Sultan Kaba*, Murat Dogan, Nesrin Ceylan, Keziban Bulan, Nihat Demir, Sekibe Zehra Dogan, \\ Selami Kocaman
}

\begin{abstract}
In present case report, four-months-old boy who referred to our pediatric endocrinology outpatient clinic from department of ophthalmology due to evaluation of endocrine and metabolic disorders for cataract was discussed. The characterized features of patient were hypotrichosis, microphthalmia, nystagmus, strabismus, congenital cataract, beaked nose, micrognathia, scaphocephaly, frontal and parietal bossing. The case has typical dysmorphic physical examination findings that appropriate diagnostic features to rare Hallermann-Streiff syndrome.
\end{abstract}

Keywords: Hallermann-Streiff syndrome, congenital cataract, microphthalmia, hypotrichosis

\section{Introduction}

Hallermann-Streiff syndrome (HSS) is characterized by a typical skull shape (brachycephaly with frontal bossing), hypotrichosis, microphthalmia, cataracts, beaked nose, micrognathia, skin atrophy, dental anomalies, and proportionate short stature $(1,2)$.

The pathogenesis of the HSS is uncertain but it is thought to be a developmental abnormality occurring at 5-7 weeks gestation. The inheritance mode has not fully understood. Although familial cases have been reported, the disease is seen mostly sporadically. Reports of patients of consanguineous or affected siblings and the recurrent abortions of the mother in cases may suggest an autosomal recessive nature in HSS. An autosomal dominant $(\mathrm{AD})$ inheritance with variable expression or new mutation has also been mentioned.

\section{Case}

A four-months-old-boy was brought with the complaint of cataract. He is the first child of an un-consanguineous marriage which the father was 25 years old and the mother was 28 years old. No problem had been observed during pregnancy and he was born with normal weight and height. It was learned that hypotrichosis and strabismus had been recognized by parent recently. The parents were healthy and they did not have any eye disorder. On physical examination, height, weight and head circumference were determined as $60.8 \mathrm{~cm}$
(10 percentile), $6.3 \mathrm{~kg}(10-25$ percentile) and $42 \mathrm{~cm}(50-75$ percentile), respectively. All of these measurements were within the normal ranges. The patient had also brachycephaly with frontal bossing, markedly hypotrichosis on scalp (like alopecia subtotalis), sparse eyebrows and eyelashes, small face, beaked nose, micrognathia, microphthalmia, bilateral cataract, strabismus and nystagmus (Figure 1, 2). The patient's interest, eye contact, response to stimuli and muscle tone was normal. Other system examinations were normal. On laboratory analyses, hemoglobin, hematocrit, platelet and white blood cell count were normal. Additionally, liver and kidney function tests, serum electrolytes, insulin like growth factor I, insulin like growth factor binding protein 3 levels were also normal. He was euthyroid, and tandem mass spectrophotometer for inborn error of metabolism (especially galactosemia, biotinidase deficiency) was found to be normal. While no abnormality was detected on whole body X-ray bone examination, magnetic resonance imaging, urinary ultrasonography, and echocardiographic examinations were also revealed normal findings. Peripheral blood karyotype analysis revealed 46, XY karyotype without any abnormality.

Informed consent for participation in the study was obtained from the parents of the subject according to the guidelines of the institutional review boards for human subjects at the participating study centers.

Received: 29-02-2016, Accepted: 15-03-2016

Yuzuncu Y1l University Faculty of Medicine, Department of Pediatrics, Van-Turkey

*Corresponding Author: Sultan Kaba E-mail: sultan33kaba@hotmail.com 


\section{Discussion}

HSS was described firstly by Hallermann and following Streiff as presence of dyscephalia, bird-like face, congenital cataracts and microphthalmia togetherness $(1,2)$. After them, Francoiss was reported a case with HSS who had skin atrophy, hypotrichosis, dental anomalies and short stature in addition to the characterized findings (3). Then, another case report was published by Steele and Bass (4) at 1970 about a HSS case associated with hypoplasia of costae and clavicle, absence of mandibular angle. But, all of these reports, it was emphasized

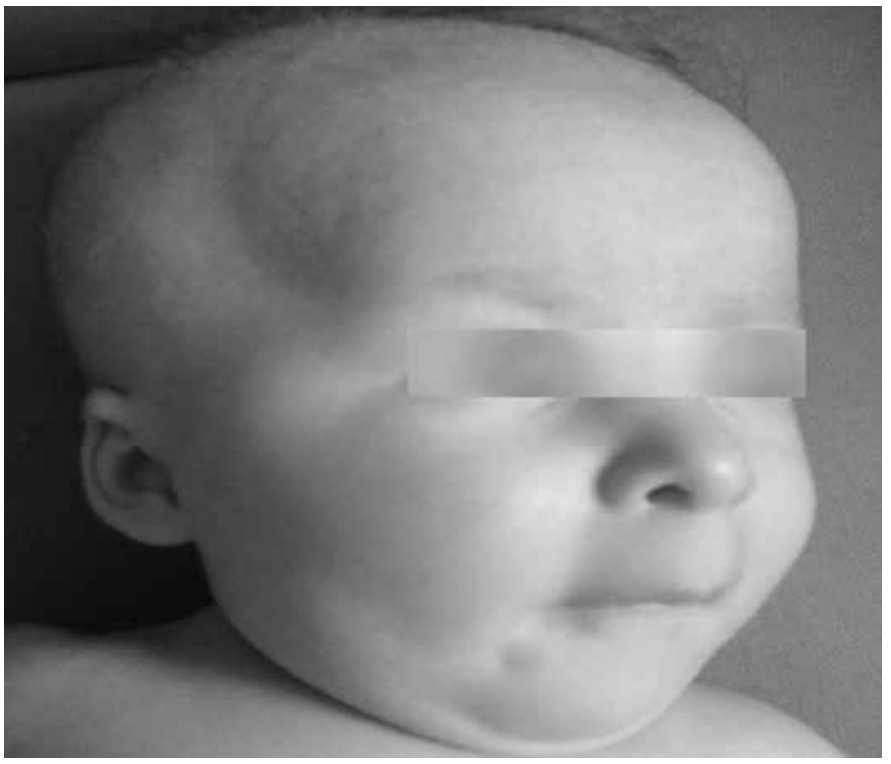

Figure 1. Brachycephaly with frontal bossing, markedly hypotrichosis on scalp, sparse eyebrows and eyelashes, small face, beaked nose, micrognathia, microphthalmia

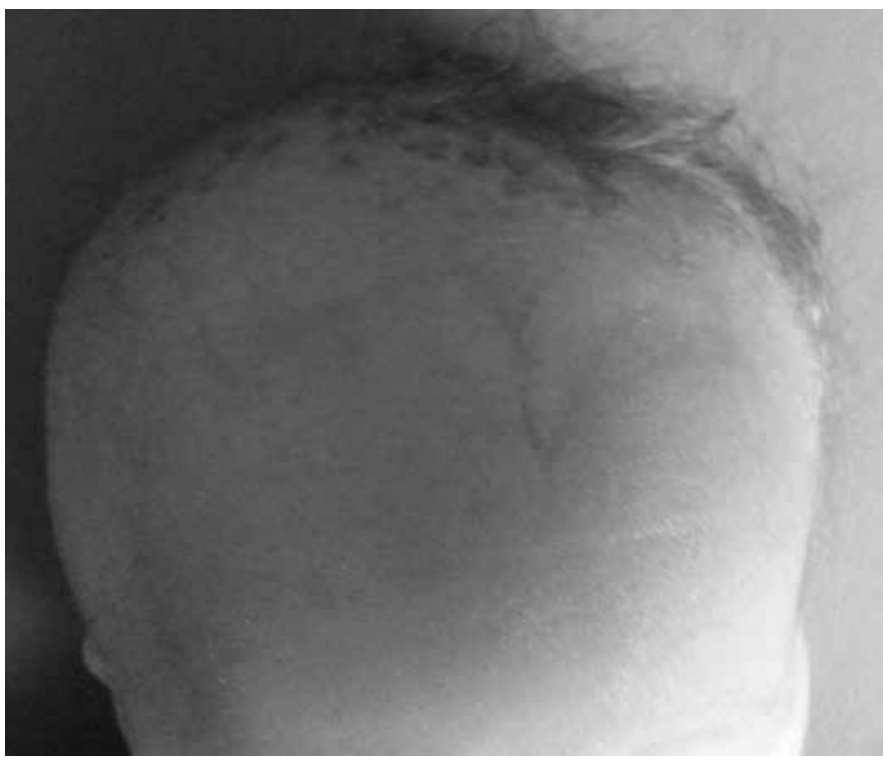

Figure 2. Markedly hypotrichosis on scalp that the diagnosis of HSS met with skepticism in the absence microphthalmia and cataract (5).

In present case, we did not speculate any dental anomalies due to younger age of our patient. Skin atrophy, short stature and bone abnormality was not detected. But the patient had brachycephaly with frontal bossing, markedly hypotrichosis on scalp, eyebrows and eyelashes, small face, beaked nose, micrognathia, microphthalmia, bilateral cataract, strabismus and nystagmus. These features were also the characterized findings of HSS. In the literature there are a lot of report with HSS without skin atrophy, bone abnormality and short stature in the literature (6).

Newborns and infants with HSS are generally suffered from upper respiratory tract obstruction and recurrent infection $(7,8)$. Association of congenital heart disease, choanal atresia, small cerebellum, very low insulin-like growth factor I level, hypothyroidism, generalized organic aciduria, has also rarely been reported in HSS (9). Mental retardation was observed to be present only in a minority of cases (4), and some authors even postulated that the absence of the neuropsychological deficit distinguished this condition from other related conditions (10). Mental and motor developments in our case were consistent with his age.

As HSS and oculodentodigital dysplasia (ODDD) share several phenotypic characteristic. Although HSS and ODDD resemble to each other in terms of typical facial appearance, dyscephaly, congenital cataract and microphthalmia which are known to be characteristic features of HSS, have been rarely reported and aren't typical in ODDD. Congenital cataract, microphthalmia, nystagmus and strabismus are the features reported in HSS while microphthalmia, microcornea and glaucoma are the most common ocular findings in ODDD. However, hypotrichosis is one of the characteristic features of HSS while it has been rarely reported in ODDD. The findings that association of dyscephaly, cataract and hypotrichosis and lack of finger anomaly as well as being a sporadic case with normal karyotype analysis decrease the likelihood ODDD in our case.

The case whose typical dysmorphic physical examinations findings appropriate diagnostic features of HSS was presented due to rarely causes of hypotrichosis and cataract.

\section{References}

1. Hallermannn W. Vogelgesicht und cataractacongenita. Klin Monatsbl Augenheilkd. 1948;113:315-318.

2. Streiff EB. Mandibulofacial dysmorphia with ocular abnormalitie. Ophthalmologica. 1950;120:79-83. 
3. François J, Victoria-Troncoso V. François' dyscephalic syndrome and skin manifestations. Ophthalmologica. 1981;183:63-67.

4. Steele RW, Bass JW. Hallermann-Streiff syndrome. Clinical and prognostic considerations. Am J Dis Child. 1970;120:462-465.

5. Warburg, M. Personal Communication. Copenhagen, Denmark 1971.

6. Grattan CE, Liddle BJ, Willshaw HE. Atrophic alopecia in the Hallermann-Strieff syndrome. Clin Exp Dermatol. 1989;14:250252.
7. Cohen MM Jr. Hallermann-Streiff syndrome: A review. Am J Med Genet. 1991;41:488-499.

8. Robinow M. Respiratory obstruction and corpulmonale in the Hallermann-Streiff syndrome. Am J Med Genet. 1991;41:515-516.

9. Hou JW. Hallermann-Streiff syndrome associated with small cerebellum, endocrinopathy and increased chromosomal breakage. Acta Paediatr. 2003;92:869-871.

10. Neki AS. Hallermann-Streiff syndrome. Indian J Ophthalmol. 1993;41:83-84. 